\title{
PREVALENCE OF AND RISK FACTORS ASSOCIATED WITH ALCOHOL ABUSE IN MOSHI, NORTHERN TANZANIA
}

\author{
TISHA MITSUNAGA* AND ULLA LARSEN† \\ *World Health Organization, Geneva, Switzerland and $\uparrow$ Department of Sociology, \\ University of Maryland, USA
}

\begin{abstract}
Summary. This study aimed to assess the prevalence of and risk factors associated with alcohol abuse among women and men in Moshi in northern Tanzania. Alcohol abuse was measured by a CAGE score of 2-4, versus 0-1 for no alcohol abuse (Ewing, 1984). Crude and adjusted logistic regression models determined odds ratios (OR) and 95\% confidence intervals (95\% CI) of alcohol abuse by characteristics of, respectively, women with partners $(n=1200)$, women without partners $(n=614)$ and men $(n=788)$ (women's partners). Prevalence of alcohol abuse was 7.0\% (95\% CI: 5.6-8.4) among women with partners, 9.3\% (95\% CI: 7.0-11.6) among women without partners, and more than double among men at 22.8\% (95\% CI: 19.9-25.8). In general, Christians had higher alcohol abuse than Muslims or other religions, as did Chagga men compared with men of other ethnic groups. Other socio-demographic characteristics, such as education or income, were not significant. Sexual behaviours were significant predictors of alcohol abuse. For example, women without partners who reported more than two partners in the last year had higher alcohol abuse compared with women reporting no partners $(\mathrm{OR}=8 \cdot 75 ; 95 \% \mathrm{CI}: 2 \cdot 37-32 \cdot 31)$, as did men reporting it is ' $\mathrm{OK}$ to hit a partner' for any reason $(\mathrm{OR}=1 \cdot 79 ; 95 \% \mathrm{CI}$ : $1 \cdot 16-2 \cdot 77)$ compared with men who did not. HIV-1 infection was not significantly associated with alcohol abuse by women or men. The Christian Church in Moshi should consider raising awareness about the harmful effects of high alcohol use among its adherents. Comprehensive programmes focusing on reducing number of partners and alcohol use, particularly by men, are needed in this community.
\end{abstract}

\section{Introduction}

Alcohol use is associated with individual mortality and morbidity from a range of mental and physical illnesses (Room, 2002; WHO, 2002). Ezzati et al. (2002) have found that alcohol consumption is one of the leading causes of the global burden of disease in 2000 , responsible for $4.0 \%$ of total disability-adjusted life years (DALYs) 
lost and 1.8 million deaths globally. In addition, individual alcohol use may have adverse social effects on family life, in the workplace, and on society in general (Secretary of Health and Human Services, 2000; Room, 2002). While per capita alcohol consumption has decreased steadily since the 1980s in the developed world, the opposite is true in developing countries (WHO, 2001; Room, 2002). In fact, Room (2002) suggests that the level of economic development is positively associated with the prevalence of alcohol consumption in developing countries. That is, higher incomes generally lead to higher consumption.

Alcohol use and abuse are well-documented in developed countries, with fewer studies looking at alcohol consumption in developing countries, and in particular in sub-Saharan Africa (Siegfried et al., 2001; Luginaah \& Dakubo, 2003). Research carried out in sub-Saharan Africa tends to focus on adolescent alcohol use (Fatoye, 2003; Luginaah \& Dakubo, 2003). Several studies have examined alcohol consumption in Tanzania. Kilonzo and his colleagues reported findings about alcohol consumption and substance misuse for all of Tanzania (Kilonzo \& Maselle, 1986; Kilonzo, 1992). Rijken et al. (1998) looked at alcohol consumption among a rural population in Mwanza, Tanzania. These authors reported that 55\% of men and 33\% of women had consumed alcohol at least once in the past year, with men reporting higher frequency and quantity of alcohol consumption than women. Overall, these figures were found to be low in comparison to estimates from other parts of Tanzania. Carlson (1992) conducted an ethnographic study of alcohol use among the Haya of Bukoba district, Tanzania, and described and analysed the way in which the Haya view excessive drinking and methods used to address drunkenness as a health problem. Kaaya et al. (1992) found the prevalence of alcohol use to be roughly $39 \%$ among secondary students in Dar es Salaam, with more than half reporting awareness of associated negative health outcomes. Kilonzo et al. (2004) investigated patterns of alcohol consumption around Dar es Salaam. Henricson (1992) looked at alcohol consumption in the Kilimanjaro area. Kapiga et al. (2007) analysed alcohol use among female bar and hotel workers in Moshi in the Kilimanjaro region. The latter study found that more than $70 \%$ report ever drinking alcohol, with a $35 \%$ prevalence of alcohol abuse based on a CAGE score of 2-4.

A number of papers cite alcohol use as a risk factor for negative health outcomes in Tanzanian populations, such as HIV infection (Ao et al., 2006; Sa \& Larsen, 2007; Kapiga et al., 2002, 2007), herpes simplex virus type 2 (HSV-2) (Kapiga et al., 2003), hypertension (Edwards et al., 2000), and injuries (Kilonzo, 1990; Nikander et al., 1992). In other sub-Saharan African countries, alcohol use is also associated with sexual abuse (Koenig et al., 2004), domestic violence (Jewkes, 2002; Koenig et al., 2003; Jewkes et al., 2003) and road traffic accidents (Odero \& Zwi, 1997; Odero et al., 1997; Odero, 1998; Andrews et al., 1999). In addition, Shayo et al. (2000) described the potential for home-brewed alcohol to contain harmful levels of bacteria.

While there are many problems linked to alcohol consumption, there may also be some health benefits associated with its use. For example, opaque beer such as orubusi, which is traditionally brewed from bananas in north-west Tanzania, contributes essential nutrients such as vitamin B, minerals, proteins and energy (Shayo et al., 2000). Further, low-to-moderate alcohol consumption may be protective for cardiovascular disease (Secretary of Health and Human Services, 2000; Room, 
2002; WHO, 2002; Suter, 2004). Finally, alcohol is often used to relieve stress or for social well-being, and it may be seen as being beneficial for one's health if taken in moderation; however, the relationship between the consumption of alcohol as a coping mechanism is complex (Secretary of Health and Human Services, 2000).

Several studies indicate that alcohol use by men is much more accepted than by women, and therefore men are more likely to consume alcohol than women in many developing countries (WHO, 2001). For example, in Lesotho, though women are the traditional brewers of beer, society strongly disapproves of their consumption of it (Mphi, 1994). In Tanzania, according to Henricson (1992), women are traditionally only permitted to drink beer on particular occasions, and certainly not in public houses. More recently, however, anecdotal evidence suggests that an increasing number of women consume alcohol outside the home. While gender differences in alcohol consumption may exist within certain communities, research shows that there are physiological differences between men and women that affect their sensitivity to alcohol (Ely et al., 1999). For example, Ely et al. (1999) found that women are more likely to have a drinking problem for the same amount of alcohol consumption as men, which may be due to differences in metabolism rates or volume of body water.

Results from the few studies that have looked at predictors of alcohol use in sub-Saharan Africa show that occupation, marital status, age and tobacco use may be associated with alcohol use among men, while female predictors include marital and employment status and education (Mphi, 1994; Rijken et al., 1998; Perdrix et al., 1999; Siegfried et al., 2001). Luginaah \& Dakubo (2003) found that men in the Upper West region of Ghana drink mainly for coping reasons, whereas women drink socially.

Tanzania is a heterogeneous country, consisting of more than 130 ethnic groups, with different languages and cultures (CIA, 2004). Presently, traditionally brewed beer (pombe) makes up roughly $90 \%$ of all alcohol consumed in Tanzania (WHO, 2001). The different ethnic groups within Tanzania grow various crops throughout the year based on geographic and climatic conditions that influence their production of traditional brew (Tusekwa et al., 2000; Kilonzo et al., 2004). For example, the Chagga use bananas (mbege), while the Pare generally use sugar cane (denegelua) (Kilonzo et al., 2004). Further, the production process is changing with the introduction of additives that accelerate fermentation (Kilonzo, 1990).

This paper focuses on alcohol consumption among the population of Moshi urban district (Moshi) in the Kilimanjaro region of Northern Tanzania. The Kilimanjaro region has traditionally been inhabited by members of the Chagga ethnic group, whose use and misuse of alcohol is an essential part of life (Maula, 1990; Setel, 1999). According to a study conducted in the late 1980s, the Kilimanjaro region has one of the highest levels of alcohol consumption in Tanzania (Henricson, 1992). Further, alcohol dependence as a disease is not generally acknowledged, in part because of the integration of alcohol in all aspects of social life in many traditional sub-Saharan African settings, as well as the ability to control intoxication through customs and social pressure (Carlson, 1992; Henricson, 1992; Kilonzo et al., 2004). For example, alcohol plays an important part in maintaining the social rubric within and across communities, and is used as a form of payment to agricultural workers (McCall, 1996). Today, alcohol is often used as a part of the brideprice. 
Due to population growth, the increased value of arable land used for coffee and banana cultivation, and Western influences, there has been substantial migration since the early 1900s from rural areas in the Kilimanjaro region and neighbouring regions to Moshi and other urban centres by both men and women (Setel, 1999). With the out-migration from rural areas and subsequent exposure to colonial institutions, socio-cultural traditions of the once rural population have adapted to the changing environments. For instance, during the economic crisis in the mid-1980s, it was not uncommon for people to supplement their income with money from brewing alcohol (Henricson, 1992). Kilonzo (1990) also suggests that production and consumption of alcohol are being carried out more and more for recreational and economic reasons rather than religious or ceremonial purposes. In addition, a pattern of binge drinking has been observed in East Africa (Kilonzo et al., 2004). It is apparent that patterns and practices of alcohol use among the population of northern Tanzania have changed and are still changing with these social, cultural and economic trends. Given the relatively high prevalence of alcohol consumption in the Kilimanjaro region, the changing patterns of alcohol use, and the negative health and social outcomes associated with it, it is important to quantify the extent to which alcohol is currently being used, and to identify risk factors associated with it in order to influence programmes and policies addressing the use and abuse of alcohol in this community. The objective of this paper was to document the prevalence of and risk factors associated with alcohol abuse (as defined below) among women and their regular partners in Moshi in the Kilimanjaro region of northern Tanzania.

\section{Methods}

\section{Community-based survey}

This study used data from a community-based survey that was conducted from November 2002 to March 2003 in Moshi. The sample was selected using a two-stage cluster design developed by the Tanzania National Bureau of Statistics. First, 150 clusters were selected proportional to the number of women aged 20-44 from the 2002 census enumeration areas. A sample of eighteen households was then selected using systematic random sampling from each of the clusters in which all women aged 20-44 were eligible to be interviewed. Men who were listed as husbands or partners of these women were also eligible for individual interviews. Information was collected on fertility, infertility, family planning, marriage, sexual practices, symptoms of sexually transmitted diseases (STDs), socio-demographic characteristics, and husband-wife relations including domestic violence and alcohol use (Larsen et al., 2007). In addition, blood and urine specimens were collected and tested for several STDs. The study protocol was approved by the Kilimanjaro Christian Medical Center (KCMC), the Tanzania National Institute for Medical Research, and the Institutional Review Boards of Harvard School of Public Health, University of Maryland, and Centers for Disease Control and Prevention.

The dependent variable, alcohol abuse, was measured by the CAGE questionnaire with yes/no answers to the following questions: (1) Have you ever felt you should Cut down on your drinking? (2) Have people Annoyed you by criticizing your drinking? (3) 
Have you ever felt bad or Guilty about your drinking? (4) Have you ever had a drink first thing in the morning to steady your nerves or to get rid of a hangover (Eye-opener)? The CAGE questionnaire is a validated assessment of alcohol abuse that is generally used as a clinical screening tool in primary care settings (Ewing, 1984). The CAGE score is defined as the number of affirmative answers to these questions ranging from zero to four. The cut-off boundary is site-specific, based on the characteristics of the environment in which it is used. In this study, a score of two or more marked a high likelihood of alcohol abuse. The dependent variable was binary: unlikely to have a drinking problem (CAGE score $0-1$ ) or likely to have a drinking problem (alcohol abuse) (CAGE score 2-4).

The men, as described above, were partners of the women surveyed. Thus, the women were stratified: with partner (currently married or living with a man and $n=1200$ ) or without partner (widowed, divorced, separated or never been married or lived with a man and $n=614)$ in order to match the analysis of the male partners $(n=788)$.

Independent variables and possible confounders identified through the literature and theoretical reasoning were broken down into four categories: socio-demographic characteristics including age at survey, ethnic group, religion and education; sexual practices including first sexual experience (women only), first sexual partner and number of partners in last year; partner behaviour, including self-reported partner CAGE score (women only), a violence indicator (women only) and believing it is OK to hit one's partner (men only); and presence of STDs including currently having STD symptoms, laboratory confirmed HIV-1, HSV-2 and Trichomonas vaginalis (T. vaginalis).

Ethnic group was categorized as Chagga and 'other', which included all other ethnic groups. Religion was categorized as Christian and Muslim or other religious affiliation. Education was broken down into two categories: respondents with zero to eight years of schooling were defined as having less than secondary; and respondents with greater than eight years of schooling were defined as having secondary or higher education. Men's occupation was categorized as farmer, professional, shopkeeper or business, and all others including skilled and unskilled labour, retired, other, unemployed and 'missing'. Women's occupation was excluded as almost half (41\%) of the women did not provide information about occupation. Men's average monthly income in the last year was categorized into six groups and was reported in Tanzanian shillings (Tsh). This variable was not captured in the women's survey as the women's income very much depended on the time of year (e.g. many women had relatively higher incomes in December because of sales and other income-generating activities related to Christmas).

Women's first sexual experience was categorized as consensual (the woman wanted to have sex), non-consensual but unforced (the woman did not want to have sex, but had it anyway) and forced (the woman did not want to have sex but was forced to have it). First sexual partner was categorized as wife/husband/cohabiting partner, regular non-cohabiting partner and 'other', which included other friend, casual acquaintance, commercial sex customer, relative and other. The variable 'ever had forced sex' was taken from the Sexual Experiences Survey (Koss \& Oros, 1982): 'Within your present relationship/outside of your present relationship have you ever had sexual intercourse when you didn't want to because your husband or partner threatened or used some degree of physical force to make you? (Twisting your arm, 
holding you down, etc.).' One item from the Conflict Tactics Scale (Straus, 1990) and two items from the Abuse Assessment Screen (McFarlane et al., 1992) were used to ascertain 12-month and lifetime partner abuse combining physical and psychological types of violence reported by the women. These included: being insulted or sworn at; being threatened with physical abuse; and being hit, slapped, kicked or otherwise physically hurt. A positive response to any of the three items was counted to indicate the presence of intimate partner violence. Men who reported one or more of the following reasons to justify hitting his partner were categorized as 'OK to hit partner': she does not do the housework to satisfaction; she disobeys him; she refuses sex; she asks about girlfriends; she takes a long time to get pregnant; she is childless; he suspects she is unfaithful; or he finds out she is unfaithful.

Men and women who reported currently having any one of the following STD symptoms were categorized as such: deep lower abdominal pain; abnormal genital discharge; foul smell in genital area; excessive genital secretions; swelling of lymph nodes in genital area; itching in genital area; burning pain on micturition; deep pain during intercourse; genital ulcers or open sores; and others. The Cronbach $a$ coefficient was determined for each of these last three indices. For partner violence $a=0.71$ for women with partners, $a=0.95$ for women without partners and for OK to hit partner $a=0.62$ for men. The STD symptoms had $\alpha=0.69$ for women with partners, $\alpha=0.74$ for women without partners and $\alpha=0.63$ for men. While consenting men and women were tested for several STD infections, due to the likely high correlation between STD infections, HSV-2 was included as a non-treatable STD as well as a marker for prior STD infection, and $T$. vaginalis was included as a treatable STD. HIV-1 was included in the model for theoretical reasons. The correlation between HIV-1 and HSV-2 was $0.85,0.83$ and 0.86 for women with a partner, women without a partner and men, respectively. None of the variables was substantially different in models excluding HIV-1.

\section{Laboratory analyses}

HIV-1 infection was determined using an enzyme-linked immunosorbent assay (ELISA) (Vironostika HIV Uni-Form II plus O, Organon, Boxtel, the Netherlands) and reactive samples were confirmed using a second ELISA (Murex 1·2.0, Murex Biotech Ltd, England, UK). Indeterminate or conflicting results were resolved by Western blot (Genetic Systems HIV-1 Western blot, Bio-Rad Laboratories, Redmond, WA, USA). Antibodies to HSV-2 were detected using type-specific HSV-2 enzyme immune assay (EIA) according to the manufacturer's instructions (HerpeSelect 2 ELISA, Focus Technologies, Cypress, CA, USA). HIV-1 and HSV-2 tests were based on sera, while urine was used for testing of $T$. vaginalis. $T$. vaginalis was tested for using the real-time multiplex polymerase chain reaction (M-PCR) assay (Rotor-Gene 3000, Corbett Research, Australia).

\section{Statistical analyses}

Statistical analyses were performed using the STATA version 8 statistical package (Stata Corporation, College Station, TX, USA). 
First, prevalence of ever-use of alcohol, alcohol abuse (as a $0-1$ or 2-4 category CAGE score) and frequency of alcohol use in past year were presented for women with partners, women without partners and men (partners of the women with partners).

Second, the socio-demographic characteristics, sexual practices, partner practices and presence of STDs were compared between women with partners and women without partners using a Pearson's $\chi^{2}$ test. This same comparison was carried out between women with partners and men (their male partners).

Third, the association between CAGE score (0-1 and 2-4) and socio-demographic characteristics, sexual practices, partner practices and presence of STDs was examined using a Pearson's $\chi^{2}$ test for women with partners, women without partners and men.

Fourth, logistic regression was used to calculate crude odds ratios (ORs) and 95\% confidence intervals (CIs) for all independent predictors of alcohol abuse for women with partners, women without partners and men. Next, adjusted models were calculated. The men's 'OK to hit' variable roughly approximated the women's partner violence variable. Only women gave information about first sexual experience and only women with partners provided information about the partner's CAGE score. The latter two variables were maintained in the adjusted models to preserve the information provided. These two variables did not affect the remaining variables substantively. Variables deemed important for theoretical reasons and variables associated with the CAGE score at the 0.20 level of significance were included in the adjusted models.

The risk factor analysis was replicated using frequency of alcohol use (at least once a week; at least once a month or less than once a month; never) and a three-category CAGE score $(0-1 ; 2 ; 3-4)$ as the dependent variable, both of which resulted in similar models. The binary CAGE score $(0-1 ; 2-4)$ is a validated measure of alcohol abuse (Ewing, 1984); therefore, the findings presented about risk factors of alcohol abuse in this paper were limited to models using the binary CAGE score.

\section{Results}

A total of 2019 women were interviewed. Of these, 176 women who reported never having had sex were removed from the data set as the survey instrument skipped questions relating to alcohol use and sexual risks for these respondents. Additionally, 29 women were omitted because they were missing data about the dependent or independent variables. The final data set included 1814 women $(90 \%$ of the 2019 women interviewed): 1200 women with partners and 614 women without partners. A total of 794 husbands and partners of these women were interviewed. One man was omitted due to a missing response for the dependent variable. In addition, five men were removed due to missing data for one or more independent variables analysed. The final data set included 788 men ( $99 \%$ of the men interviewed).

Prevalence of ever use of alcohol (not shown) among women with partners was $50 \cdot 8 \%(95 \%$ CI: 47.9-53.6) and slightly higher among women without partners at 57.5\% (95\% CI: 53.5-61.4). Among men, ever-use was much higher at $68 \cdot 3 \%$ (95\% CI: 64.9-71.5). While prevalence of alcohol abuse (CAGE score 2-4) was overall much 
lower, the trends between the three groups were similar: prevalence of alcohol abuse was $7 \cdot 0 \%(95 \% \mathrm{CI}: 5 \cdot 6-8 \cdot 4)$ among women with partners, 9.3\% (95\% CI: $7 \cdot 0-11 \cdot 6)$ among women without partners, and more than double among men at $22.8 \%(95 \%$ CI: 19.9-25.8). Interestingly, $21 \cdot 4 \%(95 \%$ CI: 19·1-23.7) of women reported having partners with alcohol abuse, which roughly approximates the men's own reports. Finally, with respect to frequency of drinking, 3.9\% (95\% CI: $2 \cdot 9-5 \cdot 2)$ of women with partners drank alcohol every day in the last year, compared with $4 \cdot 6 \%(95 \% \mathrm{CI}$ : $3 \cdot 1-6 \cdot 6)$ of women without partners and 15.9\% (95\% CI: 13.4-18.6) of men (not shown).

Table 1 shows characteristics of women with partners, women without partners and men. Women with and without partners were statistically different in reported age, ethnic group, religion, education, first sexual experience, first sexual partner, number of partners in last year and partner violence. Furthermore, women without partners had a higher prevalence of HIV-1 (11.2\% versus 9.4\%) and $T$. vaginalis $(10 \cdot 1 \%$ versus $9 \cdot 1 \%)$. Women with partners differed from men in the following characteristics: age, ethnic group, education, first sexual partner and number of partners in last year. In addition, $26.9 \%$ of women with partners reported partner violence and $19 \cdot 5 \%$ of men thought it acceptable to hit their partner for any reason; there was a higher prevalence of reported current STD symptoms among women with or without partners than men $(25 \cdot 8 \%, 24 \cdot 3 \%$ versus $8 \cdot 0 \%)$; and $47 \cdot 4 \%$ of women with partners compared to $39 \cdot 3 \%$ of men were HSV-2 positive.

Table 1 also presents differences in characteristics by CAGE score within the three groups. In general, there was more variability across the three groups than within each group. Among women with partners, first sexual experience, first sexual partner, partner violence and $T$. vaginalis infection were statistically different by CAGE score. A greater proportion of women who reported partner alcohol abuse (CAGE score 2-4) also reported abusing alcohol themselves compared with those reporting that their partner did not abuse alcohol (CAGE score $0-1)(17 \cdot 1 \%$ versus $4 \cdot 2 \%$ ). In addition, alcohol abuse was more prevalent among Christians as compared with Muslims or others $(9 \cdot 1 \%$ versus $3 \cdot 9 \%$ for women with a partner and $10 \cdot 2 \%$ versus $7 \cdot 2 \%$ for women without a partner).

Among women without partners, first sexual experience, number of partners and current STD symptoms were significantly different between those who abused alcohol and those who did not. For instance, a greater proportion of women who reported their first sexual experience as being forced also reported alcohol abuse as compared with women who reported their first sexual experience as being consensual $(18 \cdot 4 \%$ versus $8 \cdot 7 \%$ ).

Among men, ethnic group, religion, number of partners and $\mathrm{OK}$ to hit were statistically different between those who reported alcohol abuse and those who did not (greater proportions were Chagga, Christian, had two or more partners in the last year, and reported it was OK to hit). For all three STDs (HIV-1, HSV-2 and T. vaginalis), a greater proportion of men reporting alcohol abuse tested positive compared with those who did not.

Tables 2, 3 and 4 present results from the crude and adjusted logistic regression models for women and men by CAGE score $(0-1,2-4)$. The effects estimates were similar, but generally attenuated in the adjusted compared with crude models. 
Table 1. Comparison of characteristics between women with partners, women without partners and men and by alcohol abuse as measured by CAGE score

\begin{tabular}{|c|c|c|c|c|c|c|c|c|c|c|c|c|c|c|c|c|c|}
\hline \multirow[b]{3}{*}{ Variable } & \multicolumn{4}{|c|}{$\begin{array}{l}\text { Women with partners } \\
\qquad(N=1200)\end{array}$} & \multirow[b]{3}{*}{$p$ value $^{\mathrm{a}}$} & \multicolumn{4}{|c|}{$\begin{array}{l}\text { Women without partners } \\
\qquad(N=614)\end{array}$} & \multirow[b]{3}{*}{$p$ value $^{\mathrm{a}}$} & \multirow[b]{3}{*}{$p$ value $^{\mathrm{b}}$} & \multicolumn{4}{|c|}{ Men $(N=788)$} & \multirow[b]{3}{*}{$p$ value $^{\mathrm{a}}$} & \multirow[b]{3}{*}{$p$ value $^{\mathrm{c}}$} \\
\hline & \multicolumn{2}{|c|}{ Sample size } & \multicolumn{2}{|c|}{$\begin{array}{l}\text { CAGE } \\
\text { score 2-4 }\end{array}$} & & \multicolumn{2}{|c|}{ Sample size } & \multicolumn{2}{|c|}{$\begin{array}{l}\text { CAGE } \\
\text { score 2-4 }\end{array}$} & & & \multicolumn{2}{|c|}{ Sample size } & \multicolumn{2}{|c|}{$\begin{array}{l}\text { CAGE } \\
\text { score 2-4 }\end{array}$} & & \\
\hline & $n$ & $\%$ & $n$ & $\%$ & & $n$ & $\%$ & $n$ & $\%$ & & & $n$ & $\%$ & $n$ & $\%$ & & \\
\hline Age at survey (years) ${ }^{\mathrm{d}}$ & & & & & $0 \cdot 74$ & & & & & $0 \cdot 54$ & $<0 \cdot 0001$ & & & & & $0 \cdot 19$ & $<0.0001$ \\
\hline $25-29$ & 304 & $25 \cdot 3$ & 23 & $7 \cdot 6$ & & 155 & $25 \cdot 2$ & 19 & $12 \cdot 3$ & & & & & & & & \\
\hline $30-34$ & 280 & $23 \cdot 3$ & 21 & $7 \cdot 5$ & & 86 & $14 \cdot 0$ & 7 & $8 \cdot 1$ & & & 194 & $24 \cdot 6$ & 42 & $21 \cdot 6$ & & \\
\hline $35-39$ & 358 & $29 \cdot 8$ & 26 & $7 \cdot 3$ & & 158 & $25 \cdot 7$ & 13 & $8 \cdot 2$ & & & 168 & $21 \cdot 3$ & 35 & $20 \cdot 8$ & & \\
\hline $40-44$ & & & & & & & & & & & & 126 & $16 \cdot 0$ & 35 & $27 \cdot 8$ & & \\
\hline $45+$ & & & & & & & & & & & & 145 & $18 \cdot 4$ & 40 & $27 \cdot 6$ & & \\
\hline Ethnic group & & & & & $0 \cdot 08$ & & & & & $0 \cdot 09$ & $<0.001$ & & & & & $<0.001$ & $0 \cdot 01$ \\
\hline Chagga & 561 & $46 \cdot 8$ & 47 & $8 \cdot 4$ & & 345 & $56 \cdot 2$ & 38 & $11 \cdot 0$ & & & 322 & $40 \cdot 9$ & 95 & $29 \cdot 5$ & & \\
\hline Other & 639 & $53 \cdot 3$ & 37 & $5 \cdot 8$ & & 269 & $43 \cdot 8$ & 19 & $7 \cdot 1$ & & & 466 & $59 \cdot 1$ & 85 & $18 \cdot 2$ & & \\
\hline Christian & 715 & $59 \cdot 6$ & 65 & $9 \cdot 1$ & & 433 & $70 \cdot 5$ & 44 & $10 \cdot 2$ & & & 458 & $58 \cdot 1$ & 127 & $27 \cdot 7$ & & \\
\hline Muslim, other & 485 & $40 \cdot 4$ & 19 & $3 \cdot 9$ & & 181 & $29 \cdot 5$ & 13 & $7 \cdot 2$ & & & 330 & $41 \cdot 9$ & 53 & $16 \cdot 1$ & & \\
\hline Education $^{\mathrm{f}}$ & & & & & $0 \cdot 44$ & & & & & $0 \cdot 75$ & $0 \cdot 04$ & & & & & $0 \cdot 57$ & $0 \cdot 001$ \\
\hline Less than secondary & 916 & $76 \cdot 3$ & 67 & $7 \cdot 3$ & & 442 & $72 \cdot 0$ & 40 & $9 \cdot 0$ & & & 547 & $69 \cdot 4$ & 128 & $23 \cdot 4$ & & \\
\hline Secondary or higher & 284 & $23 \cdot 7$ & 17 & $6 \cdot 0$ & & 172 & $28 \cdot 0$ & 17 & $9 \cdot 9$ & & & 241 & $30 \cdot 6$ & 52 & $21 \cdot 6$ & & \\
\hline Occupation $^{\mathrm{g}}$ & & & & & & & & & & & & & & & & $0 \cdot 91$ & \\
\hline Farmer & & & & & & & & & & & & 115 & $14 \cdot 6$ & 26 & $22 \cdot 6$ & & \\
\hline $\begin{array}{l}\text { Professional, business, } \\
\text { shopkeeper }\end{array}$ & & & & & & & & & & & & 108 & $13 \cdot 7$ & 23 & $21 \cdot 3$ & & \\
\hline Other & & & & & & & & & & & & 565 & $71 \cdot 7$ & 131 & $23 \cdot 2$ & & \\
\hline Income (Tsh) & & & & & & & & & & & & & & & & $0 \cdot 3$ & \\
\hline 0 & & & & & & & & & & & & 44 & $5 \cdot 6$ & 12 & $27 \cdot 3$ & & \\
\hline$<30,000$ & & & & & & & & & & & & 129 & $16 \cdot 4$ & 36 & $27 \cdot 9$ & & \\
\hline $30,000-49,000$ & & & & & & & & & & & & 254 & $32 \cdot 2$ & 51 & $20 \cdot 1$ & & \\
\hline $50,000-69,000$ & & & & & & & & & & & & 163 & $20 \cdot 7$ & 31 & $19 \cdot 0$ & & \\
\hline
\end{tabular}

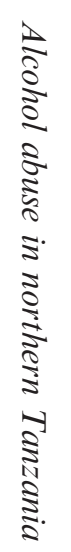


Table 1. Continued

\begin{tabular}{|c|c|c|c|c|c|c|c|c|c|c|c|c|c|c|c|c|c|}
\hline \multirow[b]{3}{*}{ Variable } & \multicolumn{4}{|c|}{$\begin{array}{l}\text { Women with partners } \\
\qquad(N=1200)\end{array}$} & \multirow[b]{3}{*}{$p$ value $^{\mathrm{a}}$} & \multicolumn{4}{|c|}{$\begin{array}{l}\text { Women without partners } \\
\qquad(N=614)\end{array}$} & \multirow[b]{3}{*}{$p$ value $^{\mathrm{a}}$} & \multirow[b]{3}{*}{$p$ value $^{\mathrm{b}}$} & \multicolumn{4}{|c|}{ Men $(N=788)$} & \multirow[b]{3}{*}{$p$ value $^{\mathrm{a}}$} & \multirow[b]{3}{*}{$p$ value } \\
\hline & \multicolumn{2}{|c|}{ Sample size } & \multicolumn{2}{|c|}{$\begin{array}{c}\text { CAGE } \\
\text { score 2-4 } \\
\end{array}$} & & \multicolumn{2}{|c|}{ Sample size } & \multicolumn{2}{|c|}{$\begin{array}{c}\text { CAGE } \\
\text { score 2-4 }\end{array}$} & & & \multicolumn{2}{|c|}{ Sample size } & \multicolumn{2}{|c|}{$\begin{array}{c}\text { CAGE } \\
\text { score 2-4 }\end{array}$} & & \\
\hline & $n$ & $\%$ & $n$ & $\%$ & & $n$ & $\%$ & $n$ & $\%$ & & & $n$ & $\%$ & $n$ & $\%$ & & \\
\hline $70,000-99,000$ & & & & & & & & & & & & 80 & $10 \cdot 2$ & 18 & $22 \cdot 5$ & & \\
\hline$\geq 100,000$ & & & & & & & & & & & & 118 & $15 \cdot 0$ & 32 & $27 \cdot 1$ & & \\
\hline First sexual experience & & & & & $0 \cdot 01$ & & & & & $0 \cdot 008$ & $0 \cdot 002$ & & & & & & \\
\hline Consensual & 925 & $77 \cdot 1$ & 54 & $5 \cdot 8$ & & 427 & $69 \cdot 5$ & 37 & $8 \cdot 7$ & & & & & & & & \\
\hline $\begin{array}{l}\text { Non-consensual, } \\
\text { unforced }\end{array}$ & 169 & $14 \cdot 1$ & 17 & $10 \cdot 1$ & & 111 & $18 \cdot 1$ & 6 & $5 \cdot 4$ & & & & & & & & \\
\hline Forced & 106 & $8 \cdot 8$ & 13 & $12 \cdot 3$ & & 76 & $12 \cdot 4$ & 14 & $18 \cdot 4$ & & & & & & & & \\
\hline First sexual partner ${ }^{\mathrm{i}}$ & & & & & $0 \cdot 001$ & & & & & $0 \cdot 80$ & $<0 \cdot 0001$ & & & & & $0 \cdot 18$ & $<0.0001$ \\
\hline $\begin{array}{l}\text { Spouse/cohabiting } \\
\text { partner }\end{array}$ & 695 & $57 \cdot 9$ & 33 & $4 \cdot 7$ & & 52 & $8 \cdot 5$ & 6 & $11 \cdot 5$ & & & 123 & $15 \cdot 6$ & 22 & $17 \cdot 9$ & & \\
\hline $\begin{array}{l}\text { Regular non-cohabiting } \\
\text { partner }\end{array}$ & 244 & $20 \cdot 3$ & 27 & $11 \cdot 1$ & & 386 & $62 \cdot 9$ & 36 & $9 \cdot 3$ & & & 162 & $20 \cdot 6$ & 33 & $20 \cdot 4$ & & \\
\hline Other & 261 & $21 \cdot 8$ & 24 & $9 \cdot 2$ & & 176 & $28 \cdot 7$ & 15 & $8 \cdot 5$ & & & 503 & $63 \cdot 8$ & 125 & $24 \cdot 9$ & & \\
\hline $\begin{array}{l}\text { Number partners in last } \\
\text { year }\end{array}$ & & & & & $0 \cdot 68$ & & & & & $0 \cdot 001$ & $<0.0001$ & & & & & $<0 \cdot 0001$ & $<0.0001$ \\
\hline None & 16 & $1 \cdot 3$ & 1 & $6 \cdot 3$ & & 223 & $36 \cdot 3$ & 9 & $4 \cdot 0$ & & & & & & & & \\
\hline One & 1168 & $97 \cdot 3$ & 81 & $6 \cdot 9$ & & 364 & $59 \cdot 3$ & 42 & $11 \cdot 5$ & & & 691 & $87 \cdot 7$ & 142 & $20 \cdot 5$ & & \\
\hline Two or more & 16 & $1 \cdot 3$ & 2 & $12 \cdot 5$ & & 27 & $4 \cdot 4$ & 6 & $22 \cdot 2$ & & & 97 & $12 \cdot 3$ & 38 & $39 \cdot 2$ & & \\
\hline Partner's CAGE score & & & & & $<\cdot 0001$ & & & & & & & & & & & & \\
\hline $0-1$ & 943 & $78 \cdot 6$ & 40 & $4 \cdot 2$ & & & & & & & & & & & & & \\
\hline $2-4$ & 257 & $21 \cdot 4$ & 44 & $17 \cdot 1$ & & & & & & & & & & & & & \\
\hline Partner violence/OK to hit ${ }^{\mathrm{i}}$ & & & & & $<\cdot 0001$ & & & & & $0 \cdot 45$ & $<0 \cdot 0001$ & & & & & $0 \cdot 02$ & $<0.0001$ \\
\hline No & 877 & $73 \cdot 1$ & 46 & $5 \cdot 5$ & & 180 & $29 \cdot 3$ & 20 & $11 \cdot 1$ & & & 634 & $80 \cdot 5$ & 134 & $21 \cdot 1$ & & \\
\hline Yes & 323 & $26 \cdot 9$ & 38 & $13 \cdot 3$ & & 128 & $20 \cdot 8$ & 13 & $10 \cdot 2$ & & & 154 & $19 \cdot 5$ & 46 & $29 \cdot 9$ & & \\
\hline Missing & & & & & & 306 & $49 \cdot 8$ & 24 & $7 \cdot 8$ & & & & & & & & \\
\hline $\begin{array}{l}\text { Currently have STD } \\
\text { symptoms }\end{array}$ & & & & & $0 \cdot 27$ & & & & & $0 \cdot 001$ & $0 \cdot 47$ & & & & & $0 \cdot 15$ & $<0.0001$ \\
\hline Yes & 310 & $25 \cdot 8$ & 26 & $8 \cdot 4$ & & 149 & $24 \cdot 3$ & 24 & $16 \cdot 1$ & & & 63 & $8 \cdot 0$ & 19 & $30 \cdot 2$ & & \\
\hline
\end{tabular}


Table 1. Continued

\begin{tabular}{|c|c|c|c|c|c|c|c|c|c|c|c|c|c|c|c|c|c|}
\hline \multirow[b]{3}{*}{ Variable } & \multicolumn{4}{|c|}{$\begin{array}{l}\text { Women with partners } \\
\qquad(N=1200)\end{array}$} & \multirow[b]{3}{*}{$p$ value $^{\mathrm{a}}$} & \multicolumn{4}{|c|}{$\begin{array}{l}\text { Women without partners } \\
\qquad(N=614)\end{array}$} & \multirow[b]{3}{*}{$p$ value $^{\mathrm{a}}$} & \multirow[b]{3}{*}{$p$ value $^{\mathrm{b}}$} & \multicolumn{4}{|c|}{$\operatorname{Men}(N=788)$} & \multirow[b]{3}{*}{$p$ value $^{\mathrm{a}}$} & \multirow[b]{3}{*}{$p$ value $^{\mathrm{c}}$} \\
\hline & \multicolumn{2}{|c|}{ Sample size } & \multicolumn{2}{|c|}{$\begin{array}{l}\text { CAGE } \\
\text { score } 2-4\end{array}$} & & \multicolumn{2}{|c|}{ Sample size } & \multicolumn{2}{|c|}{$\begin{array}{l}\text { CAGE } \\
\text { score } 2-4\end{array}$} & & & \multicolumn{2}{|c|}{ Sample size } & \multicolumn{2}{|c|}{$\begin{array}{l}\text { CAGE } \\
\text { score 2-4 }\end{array}$} & & \\
\hline & $n$ & $\%$ & $n$ & $\%$ & & $n$ & $\%$ & $n$ & $\%$ & & & $n$ & $\%$ & $n$ & $\%$ & & \\
\hline No & 890 & $74 \cdot 2$ & 58 & $6 \cdot 5$ & & 465 & $75 \cdot 7$ & 33 & $7 \cdot 1$ & & & 725 & $92 \cdot 0$ & 161 & $22 \cdot 2$ & & \\
\hline HIV-1 status & & & & & $0 \cdot 39$ & & & & & $0 \cdot 85$ & $0 \cdot 002$ & & & & & $0 \cdot 004$ & $0 \cdot 16$ \\
\hline Positive & 79 & $9 \cdot 4$ & 8 & $10 \cdot 1$ & & 69 & $11 \cdot 2$ & 7 & $10 \cdot 1$ & & & 37 & $6 \cdot 6$ & 14 & $37 \cdot 8$ & & \\
\hline Negative & 765 & $63 \cdot 8$ & 55 & $7 \cdot 2$ & & 364 & $59 \cdot 3$ & 35 & $9 \cdot 6$ & & & 525 & $66 \cdot 6$ & 129 & $24 \cdot 6$ & & \\
\hline Not tested & 356 & $29 \cdot 7$ & 21 & $5 \cdot 9$ & & 181 & $29 \cdot 5$ & 15 & $8 \cdot 3$ & & & 226 & $28 \cdot 7$ & 37 & $16 \cdot 4$ & & \\
\hline HSV-2 status & & & & & $0 \cdot 62$ & & & & & $0 \cdot 72$ & $0 \cdot 40$ & & & & & $0 \cdot 001$ & $0 \cdot 009$ \\
\hline Positive & 400 & $47 \cdot 4$ & 30 & $7 \cdot 5$ & & 188 & $30 \cdot 6$ & 20 & $10 \cdot 6$ & & & 221 & $39 \cdot 3$ & 68 & $30 \cdot 8$ & & \\
\hline Negative & 444 & $37 \cdot 0$ & 33 & $7 \cdot 4$ & & 245 & $39 \cdot 9$ & 22 & $9 \cdot 0$ & & & 342 & $43 \cdot 4$ & 75 & $21 \cdot 9$ & & \\
\hline Not tested & 356 & $29 \cdot 7$ & 21 & $5 \cdot 9$ & & 181 & $29 \cdot 5$ & 15 & $8 \cdot 3$ & & & 225 & $28 \cdot 6$ & 37 & $16 \cdot 4$ & & \\
\hline T. vaginalis & & & & & $0 \cdot 04$ & & & & & $0 \cdot 08$ & $0 \cdot 02$ & & & & & $<0 \cdot 0001$ & $0 \cdot 14$ \\
\hline Yes & 79 & $9 \cdot 1$ & 10 & $12 \cdot 7$ & & 62 & $10 \cdot 1$ & 9 & $4 \cdot 5$ & & & 38 & $6 \cdot 5$ & 17 & $44 \cdot 7$ & & \\
\hline Not tested & 332 & $27 \cdot 7$ & 16 & $4 \cdot 8$ & & 175 & $28 \cdot 5$ & 10 & $5 \cdot 7$ & & & 204 & $25 \cdot 9$ & 33 & $16 \cdot 2$ & & \\
\hline Total & 1200 & & 84 & $7 \cdot 0$ & & 614 & & 57 & $9 \cdot 3$ & & & 788 & & 180 & $22 \cdot 8$ & & \\
\hline
\end{tabular}

apearson's $\chi^{2}$ test comparing CAGE score $0-1$ versus $2-4$

'Pearson's $\chi^{2}$ test comparing characteristics between women with partners and women without partners.

'Pearson's $\chi^{2}$ test comparing characteristics between women with partners and men.

dAge categories for women: 20-24; 25-29; 30-34; 35-44. Age categories for men: 20-29; 30-34; 35-39; 40-44; 45+.

${ }^{\mathrm{e}}$ Muslim and other for women with partners: Muslim $(n=431)$, none $(n=3)$, other $(n=51)$; women without partners: Muslim $(n=165)$, none $(n=1)$, other $(n=15)$; Men: Muslim $(n=287)$, none $(n=2)$, other $(n=41)$

Secondary or higher education refers to having completed $9+$ years of schooling.

gther includes skilled and unskilled labour, retired, other, unemployed and missing.

Other includes other friend, casual acquaintance, commercial sex customer, relative and other.

'Men's OK to hit variable (yes/no) roughly approximates women's partner violence variable.

${ }^{j}$ STD symptoms include: deep lower abdominal pain, abnormal genital discharge, foul smell in genital area, excessive genital secretions, swelling of lymph

nodes in genital area, itching in genital area, burning pain on micturition, deep pain during intercourse, genital ulcers or open sores, and others. 
Table 2. Crude and adjusted odds ratios (ORs) and 95\% confidence intervals (CIs) for characteristics associated with alcohol abuse for women with partners $(N=1200)$

\begin{tabular}{|c|c|c|c|c|c|c|}
\hline \multirow[b]{2}{*}{ Variable } & \multicolumn{3}{|c|}{ Crude } & \multicolumn{3}{|c|}{ Adjusted } \\
\hline & OR & $95 \% \mathrm{CI}$ & $p$ value $^{\mathrm{a}}$ & OR & $95 \% \mathrm{CI}$ & $p$ value \\
\hline \multicolumn{7}{|l|}{ Age at survey (years) } \\
\hline $20-24$ & $1 \cdot 00$ & & & $1 \cdot 00$ & & \\
\hline $25-29$ & $1 \cdot 43$ & $0 \cdot 72-2 \cdot 83$ & $0 \cdot 31$ & $1 \cdot 20$ & $0 \cdot 59-2 \cdot 48$ & $0 \cdot 61$ \\
\hline $30-34$ & $1 \cdot 41$ & $0 \cdot 70-2 \cdot 84$ & $0 \cdot 33$ & $1 \cdot 17$ & $0 \cdot 56-2 \cdot 45$ & $0 \cdot 68$ \\
\hline $35-44$ & $1 \cdot 36$ & $0 \cdot 70-2 \cdot 67$ & $0 \cdot 36$ & $1 \cdot 18$ & $0 \cdot 57-2 \cdot 43$ & $0 \cdot 65$ \\
\hline \multicolumn{7}{|l|}{ Ethnic group } \\
\hline Chagga & $1 \cdot 00$ & & & $1 \cdot 00$ & & \\
\hline Other & $0 \cdot 67$ & $0 \cdot 43-1 \cdot 05$ & $0 \cdot 08$ & $0 \cdot 99$ & $0 \cdot 60-1 \cdot 66$ & $0 \cdot 98$ \\
\hline \multicolumn{7}{|l|}{ Religion $^{\mathrm{b}}$} \\
\hline Christian & $2 \cdot 45$ & $1 \cdot 45-4 \cdot 15$ & $0 \cdot 001$ & $1 \cdot 80$ & $0 \cdot 99-3 \cdot 26$ & $0 \cdot 05$ \\
\hline Muslim, other & $1 \cdot 00$ & & & $1 \cdot 00$ & & \\
\hline \multicolumn{7}{|l|}{ Education $^{\mathrm{c}}$} \\
\hline Less than secondary & $1 \cdot 24$ & $0 \cdot 72-2 \cdot 15$ & $0 \cdot 44$ & $1 \cdot 21$ & $0 \cdot 67-2 \cdot 19$ & $0 \cdot 53$ \\
\hline Secondary or higher & $1 \cdot 00$ & & & $1 \cdot 00$ & & \\
\hline First sexual experience & & & & & & \\
\hline Consensual & $1 \cdot 00$ & & & $1 \cdot 00$ & & \\
\hline Non-consensual, unforced & $1 \cdot 80$ & $1 \cdot 02-3 \cdot 20$ & $0 \cdot 04$ & $1 \cdot 43$ & $0 \cdot 77-2 \cdot 66$ & $0 \cdot 26$ \\
\hline Forced & $2 \cdot 25$ & $1 \cdot 19-4 \cdot 29$ & $0 \cdot 01$ & $1 \cdot 25$ & $0 \cdot 61-2 \cdot 55$ & $0 \cdot 54$ \\
\hline \multicolumn{7}{|l|}{ First sexual partner ${ }^{\mathrm{d}}$} \\
\hline Husband/cohabiting partner & $1 \cdot 00$ & & & $1 \cdot 00$ & & \\
\hline Regular non-cohabiting partner & $2 \cdot 50$ & $1 \cdot 47-4 \cdot 25$ & $0 \cdot 001$ & $2 \cdot 07$ & $1 \cdot 16-3 \cdot 70$ & $0 \cdot 01$ \\
\hline Other & $2 \cdot 03$ & $1 \cdot 18-3 \cdot 51$ & $0 \cdot 01$ & $1 \cdot 65$ & $0 \cdot 91-2 \cdot 99$ & $0 \cdot 10$ \\
\hline \multicolumn{7}{|l|}{ Number partners in last year } \\
\hline None & $1 \cdot 00$ & & & $1 \cdot 00$ & & \\
\hline One & $1 \cdot 12$ & $0 \cdot 15-8 \cdot 57$ & $0 \cdot 92$ & $1 \cdot 72$ & $0 \cdot 20-14 \cdot 50$ & $0 \cdot 62$ \\
\hline Two or more & $2 \cdot 14$ & $0 \cdot 17-26 \cdot 33$ & $0 \cdot 55$ & $1 \cdot 26$ & $0 \cdot 09-17 \cdot 02$ & $0 \cdot 86$ \\
\hline \multicolumn{7}{|l|}{ Partner's CAGE score } \\
\hline $0-1$ & $1 \cdot 00$ & & & $1 \cdot 00$ & & \\
\hline $2-4$ & $4 \cdot 66$ & $2 \cdot 96-7 \cdot 34$ & $<\cdot 0001$ & $3 \cdot 47$ & $2 \cdot 11-5 \cdot 71$ & $<0 \cdot 0001$ \\
\hline \multicolumn{7}{|l|}{ Partner violence ${ }^{\mathrm{e}}$} \\
\hline No & $1 \cdot 00$ & & & $1 \cdot 00$ & & \\
\hline Yes & $2 \cdot 40$ & $1 \cdot 53-3 \cdot 76$ & $<\cdot 0001$ & $1 \cdot 49$ & $0 \cdot 88-2 \cdot 50$ & $0 \cdot 140$ \\
\hline \multicolumn{7}{|l|}{ Currently have STD symptoms ${ }^{\mathrm{f}}$} \\
\hline Yes & $1 \cdot 31$ & $0 \cdot 81-2 \cdot 13$ & $0 \cdot 27$ & $0 \cdot 92$ & $0 \cdot 54-1 \cdot 57$ & $0 \cdot 76$ \\
\hline No & $1 \cdot 00$ & & & $1 \cdot 00$ & & \\
\hline \multicolumn{7}{|l|}{ HIV-1 status } \\
\hline Positive & $1 \cdot 45$ & $0 \cdot 67-3 \cdot 18$ & $0 \cdot 35$ & $1 \cdot 42$ & $0 \cdot 62-3 \cdot 27$ & $0 \cdot 41$ \\
\hline Negative & $1 \cdot 00$ & & & $1 \cdot 00$ & & \\
\hline Not tested & $0 \cdot 81$ & $0 \cdot 48-1 \cdot 36$ & $0 \cdot 42$ & & & \\
\hline
\end{tabular}


Table 2. Continued

\begin{tabular}{|c|c|c|c|c|c|c|}
\hline \multirow[b]{2}{*}{ Variable } & \multicolumn{3}{|c|}{ Crude } & \multicolumn{3}{|c|}{ Adjusted } \\
\hline & OR & $95 \% \mathrm{CI}$ & $p$ value $^{\mathrm{a}}$ & OR & $95 \% \mathrm{CI}$ & $p$ value \\
\hline \multicolumn{7}{|l|}{ HSV-2 status } \\
\hline Positive & $1 \cdot 01$ & $0.60-1.69$ & $0 \cdot 97$ & $0 \cdot 81$ & $0 \cdot 46-1 \cdot 42$ & $0 \cdot 46$ \\
\hline Negative & $1 \cdot 00$ & & & $1 \cdot 00$ & & \\
\hline Not tested & $0 \cdot 78$ & $0 \cdot 44-1 \cdot 37$ & $0 \cdot 39$ & $1 \cdot 55$ & $0 \cdot 66-3 \cdot 63$ & $0 \cdot 32$ \\
\hline \multicolumn{7}{|l|}{ T. vaginalis } \\
\hline Yes & $1 \cdot 83$ & $0 \cdot 89-3 \cdot 73$ & $0 \cdot 10$ & $1 \cdot 47$ & $0 \cdot 67-3 \cdot 20$ & $0 \cdot 33$ \\
\hline No & $1 \cdot 00$ & & & $1 \cdot 00$ & & \\
\hline Not tested & $0 \cdot 64$ & $0 \cdot 36-1 \cdot 13$ & $0 \cdot 12$ & $0 \cdot 56$ & $0 \cdot 23-1 \cdot 34$ & $0 \cdot 19$ \\
\hline
\end{tabular}

${ }^{\mathrm{a}}$ Wald test.

bMuslim and other: Muslim $(n=431)$, none $(n=3)$, other $(n=51)$.

${ }^{\mathrm{c}}$ Secondary or higher education refers to having completed 9+ years of schooling.

${ }^{\mathrm{d}}$ Other includes other friend, casual acquaintance, commercial sex customer, relative and other.

${ }^{\mathrm{e}}$ Defined as a positive response to any one of the following: being insulted or sworn at by a partner; being threatened with physical abuse by a partner; or being hit, slapped, kicked or otherwise physically hurt by a partner.

${ }^{\mathrm{f}}$ STD symptoms include: deep lower abdominal pain, abnormal genital discharge, foul smell in genital area, excessive genital secretions, swelling of lymph nodes in genital area, itching in genital area, burning pain on micturition, deep pain during intercourse, genital ulcers or open sores, and others.

With respect to the effects of socio-demographic characteristics, Christians had greater odds of a high CAGE score (2-4) relative to Muslims or other for women with partners $(\mathrm{OR}=1 \cdot 80 ; 95 \% \mathrm{CI}$ : 0.99-3.26) and for men $(\mathrm{OR}=1.69 ; 95 \% \mathrm{CI}$ : $1 \cdot 08-2 \cdot 62)$.

Sexual practices were highly predictive of alcohol abuse. For instance, reporting two or more partners in the last year relative to none was associated with higher alcohol abuse among women without partners (OR=8·75; 95\% CI: 2·37-32.31), while the number of partners did not matter for the group of women with partners. Men with two or more partners relative to one also had higher odds of alcohol abuse $(\mathrm{OR}=2 \cdot 37 ; 95 \% \mathrm{CI}: 1 \cdot 45-3 \cdot 86)$. HIV-1, HSV-2 and $T$. vaginalis infections were non-significant for women with and without partners, while men with $T$. vaginalis at the survey date had more than double the odds of a high CAGE score $(\mathrm{OR}=2.93$; 95\% CI: 1.42-6.03) in the adjusted model.

Finally, for women with a partner, the most important predictor of alcohol abuse was the partner's CAGE score, i.e. women whose partner scored $2-4$ on the CAGE scale were more likely to abuse alcohol themselves (OR $=3 \cdot 47 ; 95 \%$ CI: $2 \cdot 11-5 \cdot 71)$. It should be noted that in the adjusted model, controlling for partner's CAGE score and first sexual partner made the effects of partner violence and first sexual experience non-significant. 
Table 3. Crude and adjusted odds ratios (ORs) and 95\% confidence intervals (CIs) for characteristics associated with alcohol abuse for women without partners $(N=614)$

\begin{tabular}{|c|c|c|c|c|c|c|}
\hline \multirow[b]{2}{*}{ Variable } & \multicolumn{3}{|c|}{ Crude } & \multicolumn{3}{|c|}{ Adjusted } \\
\hline & OR & $95 \% \mathrm{CI}$ & $p$ value $^{\mathrm{a}}$ & OR & $95 \% \mathrm{CI}$ & $p$ value \\
\hline \multicolumn{7}{|l|}{ Age at survey (years) } \\
\hline $20-24$ & $1 \cdot 00$ & & & $1 \cdot 00$ & & \\
\hline $25-29$ & $1 \cdot 53$ & $0 \cdot 77-3 \cdot 02$ & $0 \cdot 22$ & $1 \cdot 65$ & $0 \cdot 78-3 \cdot 50$ & $0 \cdot 19$ \\
\hline $30-34$ & $0 \cdot 97$ & $0 \cdot 39-2 \cdot 41$ & $0 \cdot 95$ & $0 \cdot 95$ & $0 \cdot 34-2 \cdot 69$ & $0 \cdot 93$ \\
\hline $35-44$ & $0 \cdot 98$ & $0 \cdot 47-2 \cdot 07$ & $0 \cdot 96$ & $1 \cdot 64$ & $0.67-3.99$ & $0 \cdot 28$ \\
\hline \multicolumn{7}{|l|}{ Ethnic group } \\
\hline Chagga & $1 \cdot 00$ & & & $1 \cdot 00$ & & \\
\hline Other & $0 \cdot 61$ & $0 \cdot 35-1 \cdot 09$ & $0 \cdot 10$ & $0 \cdot 67$ & $0 \cdot 34-1 \cdot 33$ & $0 \cdot 26$ \\
\hline \multicolumn{7}{|l|}{ Religion $^{\mathrm{b}}$} \\
\hline Christian & $1 \cdot 46$ & $0 \cdot 77-2 \cdot 79$ & $0 \cdot 25$ & $1 \cdot 35$ & $0 \cdot 62-2 \cdot 94$ & $0 \cdot 44$ \\
\hline Muslim, other & $1 \cdot 00$ & & & $1 \cdot 00$ & & \\
\hline \multicolumn{7}{|l|}{ Education $^{\mathrm{c}}$} \\
\hline Less than secondary & $0 \cdot 91$ & $0 \cdot 50-1 \cdot 65$ & $0 \cdot 75$ & $0 \cdot 80$ & $0 \cdot 40-1 \cdot 60$ & $0 \cdot 53$ \\
\hline Secondary or higher & $1 \cdot 00$ & & & $1 \cdot 00$ & & \\
\hline \multicolumn{7}{|l|}{ First sexual experience } \\
\hline Consensual & $1 \cdot 00$ & & & $1 \cdot 00$ & & \\
\hline Non-consensual, unforced & $0 \cdot 60$ & $0 \cdot 25-1 \cdot 47$ & $0 \cdot 26$ & $0 \cdot 67$ & $0 \cdot 27-1 \cdot 69$ & $0 \cdot 4$ \\
\hline Forced & $2 \cdot 38$ & $1 \cdot 22-4 \cdot 66$ & $0 \cdot 01$ & 1.99 & $0 \cdot 93-4 \cdot 24$ & $0 \cdot 08$ \\
\hline \multicolumn{7}{|l|}{ First sexual partner ${ }^{\mathrm{d}}$} \\
\hline Husband/cohabiting partner & $1 \cdot 00$ & & & $1 \cdot 00$ & & \\
\hline Regular non-cohabiting partner & $0 \cdot 79$ & $0 \cdot 32-1 \cdot 97$ & $0 \cdot 61$ & $0 \cdot 69$ & $0 \cdot 25-1 \cdot 93$ & $0 \cdot 49$ \\
\hline Other & $0 \cdot 71$ & $0 \cdot 26-1 \cdot 95$ & $0 \cdot 51$ & $0 \cdot 64$ & $0 \cdot 21-1 \cdot 92$ & $0 \cdot 42$ \\
\hline \multicolumn{7}{|l|}{ Number partners in last year } \\
\hline None & $1 \cdot 00$ & & & $1 \cdot 00$ & & \\
\hline One & $3 \cdot 10$ & $1 \cdot 48-6 \cdot 50$ & 0.003 & $3 \cdot 38$ & $1 \cdot 49-7 \cdot 67$ & $0 \cdot 004$ \\
\hline Two or more & $6 \cdot 79$ & $2 \cdot 20-20 \cdot 95$ & $0 \cdot 001$ & $8 \cdot 75$ & $2 \cdot 37-32 \cdot 31$ & $0 \cdot 001$ \\
\hline \multicolumn{7}{|l|}{ Partner violence ${ }^{\mathrm{e}}$} \\
\hline No & $1 \cdot 00$ & & & $1 \cdot 00$ & & \\
\hline Yes & $0 \cdot 90$ & $0 \cdot 43-1 \cdot 89$ & $0 \cdot 79$ & $1 \cdot 01$ & $0 \cdot 43-2 \cdot 35$ & $0 \cdot 98$ \\
\hline Missing & $0 \cdot 68$ & $0 \cdot 36-1 \cdot 27$ & $0 \cdot 23$ & $0 \cdot 98$ & $0 \cdot 48-1.96$ & $0 \cdot 94$ \\
\hline \multicolumn{7}{|l|}{ Currently have STD symptoms $\mathrm{s}^{\mathrm{f}}$} \\
\hline Yes & $2 \cdot 51$ & $1 \cdot 43-4 \cdot 41$ & $0 \cdot 001$ & $1 \cdot 87$ & $0 \cdot 98-3 \cdot 56$ & $0 \cdot 06$ \\
\hline No & $1 \cdot 00$ & & & $1 \cdot 00$ & & \\
\hline \multicolumn{7}{|l|}{ HIV-1 status } \\
\hline Positive & $1 \cdot 06$ & $0 \cdot 45-2 \cdot 50$ & $0 \cdot 89$ & $0 \cdot 83$ & $0 \cdot 33-2 \cdot 07$ & $0 \cdot 68$ \\
\hline Negative & $1 \cdot 00$ & & & $1 \cdot 00$ & & \\
\hline Not tested & $0 \cdot 85$ & $0 \cdot 45-1 \cdot 60$ & $0 \cdot 61$ & 1.95 & $0 \cdot 77-4.94$ & $0 \cdot 16$ \\
\hline \multicolumn{7}{|l|}{ HSV-2 status } \\
\hline Positive & $1 \cdot 21$ & $0 \cdot 64-2 \cdot 28$ & $0 \cdot 56$ & $1 \cdot 05$ & $0 \cdot 51-2 \cdot 14$ & $0 \cdot 9$ \\
\hline Negative & $1 \cdot 00$ & & & $1 \cdot 00$ & & \\
\hline Not tested & 0.92 & $0 \cdot 46-1 \cdot 82$ & $0 \cdot 80$ & & & \\
\hline
\end{tabular}


Table 3. Continued

\begin{tabular}{|c|c|c|c|c|c|c|}
\hline \multirow[b]{2}{*}{ Variable } & \multicolumn{3}{|c|}{ Crude } & \multicolumn{3}{|c|}{ Adjusted } \\
\hline & OR & $95 \% \mathrm{CI}$ & $p$ value $^{\mathrm{a}}$ & OR & $95 \% \mathrm{CI}$ & $p$ value \\
\hline \multicolumn{7}{|l|}{ T. vaginalis } \\
\hline Yes & $1 \cdot 51$ & $0 \cdot 69-3 \cdot 31$ & $0 \cdot 30$ & $1 \cdot 78$ & $0 \cdot 74-4 \cdot 28$ & $0 \cdot 2$ \\
\hline No & $1 \cdot 00$ & & & $1 \cdot 00$ & & \\
\hline Not tested & $0 \cdot 54$ & $0 \cdot 26-1 \cdot 11$ & $0 \cdot 10$ & $0 \cdot 45$ & $0 \cdot 17-1 \cdot 25$ & $0 \cdot 13$ \\
\hline
\end{tabular}

${ }^{\mathrm{a}}$ Wald test.

${ }^{\mathrm{b}}$ Muslim and other: Muslim $(n=165)$, none $(n=1)$, other $(n=15)$.

'Secondary or higher education refers to having completed $9+$ years of schooling.

${ }^{\mathrm{d}}$ Other includes other friend, casual acquaintance, commercial sex customer, relative and other.

${ }^{\mathrm{e}}$ Defined as a positive response to any one of the following: being insulted or sworn at by a partner; being threatened with physical abuse by a partner; or being hit, slapped, kicked or otherwise physically hurt by a partner.

${ }^{\mathrm{f}}$ STD symptoms include: deep lower abdominal pain, abnormal genital discharge, foul smell in genital area, excessive genital secretions, swelling of lymph nodes in genital area, itching in genital area, burning pain on micturition, deep pain during intercourse, genital ulcers or open sores, and others.

\section{Discussion}

The results of this study showed that alcohol abuse exists in the general population in Moshi. It was more prevalent among men than women, and there was no significant difference between women with and without partners. While less than $10 \%$ of women had a CAGE score of $2-4$, almost one quarter of men scored 2-4. This pattern corresponded with the ever-use of alcohol for which men reported a higher prevalence than women. However, this study involving a community-based sample indicated that the level of alcohol abuse among Moshi women was much lower than the $35 \%$ found among a group of bar and hotel workers in the same region (Kapiga et al., 2007). Women's perceptions of alcohol abuse in their male partners approximated closely what the men themselves reported. In general, Chagga men and Christian men and women with partners were more likely to report higher CAGE scores. These findings were not surprising given the history of the region. One male interviewee in a qualitative study conducted by P. Setel claims, 'Chagga are boozers'. Otherwise, socio-demographic characteristics were not related to alcohol abuse.

Education was not significantly associated with alcohol abuse (a 2-4 CAGE score). Preliminary analyses showed that occupation and income were not significantly associated with alcohol abuse among men and those variables could not be measured among women. It is therefore difficult to determine how wealth may interact with alcohol consumption and abuse, and to support the theory presented in the introduction suggesting that economic development leads to higher alcohol consumption (Room, 2002). However, Moshi is relatively well-developed compared to many other areas of Tanzania or the East African region in general, and it is possible that 
Table 4. Crude and adjusted odds ratios (ORs) and 95\% confidence intervals (CIs) for characteristics associated with alcohol abuse for men $(N=788)$

\begin{tabular}{|c|c|c|c|c|c|c|}
\hline \multirow[b]{2}{*}{ Variable } & \multicolumn{3}{|c|}{ Crude } & \multicolumn{3}{|c|}{ Adjusted } \\
\hline & OR & $95 \% \mathrm{CI}$ & $p$ value $^{\mathrm{a}}$ & OR & $95 \% \mathrm{CI}$ & $p$ value \\
\hline \multicolumn{7}{|l|}{ Age at survey (years) } \\
\hline $20-29$ & $1 \cdot 00$ & & & $1 \cdot 00$ & & \\
\hline $30-34$ & $1 \cdot 25$ & $0 \cdot 74-2 \cdot 14$ & $0 \cdot 41$ & $1 \cdot 34$ & $0 \cdot 75-2 \cdot 37$ & $0 \cdot 32$ \\
\hline $35-39$ & $1 \cdot 19$ & $0 \cdot 69-2 \cdot 08$ & $0 \cdot 53$ & $1 \cdot 21$ & $0 \cdot 67-2 \cdot 20$ & $0 \cdot 53$ \\
\hline $40-44$ & $1 \cdot 74$ & $0 \cdot 99-3 \cdot 07$ & $0 \cdot 05$ & $1 \cdot 69$ & $0 \cdot 91-3 \cdot 14$ & $0 \cdot 09$ \\
\hline $45+$ & $1 \cdot 73$ & $0.99-2.99$ & $0 \cdot 05$ & $1 \cdot 72$ & $0 \cdot 93-3 \cdot 18$ & $0 \cdot 08$ \\
\hline \multicolumn{7}{|l|}{ Ethnic group } \\
\hline Chagga & $1 \cdot 00$ & & & $1 \cdot 00$ & & \\
\hline Other & $0 \cdot 53$ & $0 \cdot 38-0 \cdot 75$ & $<0 \cdot 001$ & $0 \cdot 64$ & $0 \cdot 43-0.97$ & $0 \cdot 03$ \\
\hline \multicolumn{7}{|l|}{ Religion $^{\mathrm{b}}$} \\
\hline Christian & $2 \cdot 01$ & $1 \cdot 40-2 \cdot 87$ & $<0 \cdot 0001$ & $1 \cdot 69$ & $1 \cdot 08-2 \cdot 62$ & $0 \cdot 02$ \\
\hline Muslim, other & $1 \cdot 00$ & & & $1 \cdot 00$ & & \\
\hline \multicolumn{7}{|l|}{ Education $^{\mathrm{c}}$} \\
\hline Less than secondary & $1 \cdot 11$ & $0 \cdot 77-1 \cdot 60$ & $0 \cdot 57$ & $1 \cdot 16$ & $0 \cdot 74-1 \cdot 80$ & $0 \cdot 52$ \\
\hline Secondary or higher & $1 \cdot 00$ & & & $1 \cdot 00$ & & \\
\hline \multicolumn{7}{|l|}{ Occupation $^{\mathrm{d}}$} \\
\hline Farmer & $1 \cdot 00$ & & & $1 \cdot 00$ & & \\
\hline Professional, business, shopkeeper & 0.93 & $0.49-1.75$ & $0 \cdot 81$ & $1 \cdot 19$ & $0 \cdot 58-2 \cdot 43$ & $0 \cdot 63$ \\
\hline Other & $1 \cdot 03$ & $0.64-1.67$ & $0 \cdot 89$ & $1 \cdot 40$ & $0 \cdot 83-2 \cdot 38$ & $0 \cdot 21$ \\
\hline \multicolumn{7}{|l|}{ Income (Tsh) } \\
\hline 0 & $1 \cdot 00$ & & & $1 \cdot 00$ & & \\
\hline$<30,000$ & $1 \cdot 16$ & $0 \cdot 54-2 \cdot 48$ & $0 \cdot 70$ & $1 \cdot 28$ & $0 \cdot 55-2 \cdot 98$ & $0 \cdot 57$ \\
\hline $30,000-49,000$ & $0 \cdot 75$ & $0 \cdot 37-1 \cdot 55$ & $0 \cdot 44$ & $0 \cdot 76$ & $0 \cdot 34-1 \cdot 70$ & $0 \cdot 51$ \\
\hline $50,000-69,000$ & $0 \cdot 70$ & $0 \cdot 33-1 \cdot 51$ & $0 \cdot 37$ & $0 \cdot 73$ & $0 \cdot 31-1 \cdot 68$ & $0 \cdot 45$ \\
\hline $70,000-99,000$ & $0 \cdot 87$ & $0 \cdot 37-2 \cdot 01$ & $0 \cdot 75$ & $0 \cdot 89$ & $0 \cdot 35-2 \cdot 24$ & $0 \cdot 81$ \\
\hline$\geq 100,000$ & $1 \cdot 17$ & $0 \cdot 54-2 \cdot 53$ & $0 \cdot 69$ & $1 \cdot 38$ & $0 \cdot 57-3 \cdot 34$ & $0 \cdot 47$ \\
\hline \multicolumn{7}{|l|}{ First sexual partner ${ }^{\mathrm{e}}$} \\
\hline Wife/cohabiting partner & $1 \cdot 00$ & & & $1 \cdot 00$ & & \\
\hline Regular non-cohabiting partner & $1 \cdot 17$ & $0 \cdot 65-2 \cdot 14$ & $0 \cdot 60$ & $0 \cdot 94$ & $0 \cdot 49-1 \cdot 81$ & $0 \cdot 86$ \\
\hline Other & $1 \cdot 52$ & $0 \cdot 92-2 \cdot 51$ & $0 \cdot 10$ & $1 \cdot 31$ & $0 \cdot 76-2 \cdot 25$ & $0 \cdot 33$ \\
\hline \multicolumn{7}{|l|}{ Number partners in last year } \\
\hline One & $1 \cdot 00$ & & & $1 \cdot 00$ & & \\
\hline Two or more & $2 \cdot 49$ & $1 \cdot 59-3 \cdot 90$ & $<0 \cdot 0001$ & $2 \cdot 37$ & $1 \cdot 45-3 \cdot 86$ & $0 \cdot 001$ \\
\hline \multicolumn{7}{|l|}{ OK to hit partner ${ }^{\mathrm{f}}$} \\
\hline Yes & $1 \cdot 59$ & $1 \cdot 07-2 \cdot 36$ & $0 \cdot 02$ & $1 \cdot 79$ & $1 \cdot 16-2 \cdot 77$ & $0 \cdot 01$ \\
\hline No & $1 \cdot 00$ & & & $1 \cdot 00$ & & \\
\hline \multicolumn{7}{|l|}{ Currently have STD symptoms ${ }^{\mathrm{g}}$} \\
\hline Yes & $1 \cdot 51$ & $0 \cdot 86-2 \cdot 66$ & $0 \cdot 15$ & $1 \cdot 06$ & $0 \cdot 56-2 \cdot 00$ & $0 \cdot 86$ \\
\hline No & $1 \cdot 00$ & & & $1 \cdot 00$ & & \\
\hline \multicolumn{7}{|l|}{ HIV-1 status } \\
\hline Positive & $1 \cdot 87$ & $0 \cdot 93-3 \cdot 74$ & $0 \cdot 08$ & $1 \cdot 53$ & $0 \cdot 72-3 \cdot 28$ & $0 \cdot 27$ \\
\hline Negative & $1 \cdot 00$ & & & $1 \cdot 00$ & & \\
\hline Not tested & $0 \cdot 60$ & $0 \cdot 40-0 \cdot 90$ & $0 \cdot 01$ & $0 \cdot 86$ & $0 \cdot 42-1 \cdot 78$ & $0 \cdot 69$ \\
\hline
\end{tabular}


Table 4. Continued

\begin{tabular}{|c|c|c|c|c|c|c|}
\hline \multirow[b]{2}{*}{ Variable } & \multicolumn{3}{|c|}{ Crude } & \multicolumn{3}{|c|}{ Adjusted } \\
\hline & OR & $95 \% \mathrm{CI}$ & $p$ value $^{\mathrm{a}}$ & OR & $95 \% \mathrm{CI}$ & $p$ value \\
\hline \multicolumn{7}{|l|}{ HSV-2 status } \\
\hline Positive & $1 \cdot 58$ & $1 \cdot 08-2 \cdot 32$ & $0 \cdot 02$ & $1 \cdot 32$ & $0 \cdot 87-2 \cdot 02$ & $0 \cdot 20$ \\
\hline Negative & $1 \cdot 00$ & & & $1 \cdot 00$ & & \\
\hline Not tested & $0 \cdot 70$ & $0 \cdot 45-1 \cdot 08$ & $0 \cdot 11$ & & & \\
\hline \multicolumn{7}{|l|}{ T. vaginalis } \\
\hline Yes & $2 \cdot 59$ & $1 \cdot 33-5 \cdot 06$ & $0 \cdot 01$ & 2.93 & $1 \cdot 42-6 \cdot 03$ & $0 \cdot 004$ \\
\hline No & $1 \cdot 00$ & & & $1 \cdot 00$ & & \\
\hline Not tested & 0.62 & $0 \cdot 41-0.94$ & $0 \cdot 03$ & 0.76 & $0 \cdot 37-1 \cdot 57$ & $0 \cdot 46$ \\
\hline
\end{tabular}

${ }^{\text {a Wald test. }}$

${ }^{\mathrm{b}}$ Muslim and other: Muslim $(n=287)$, none $(n=2)$, other $(n=41)$.

${ }^{\mathrm{c}}$ Secondary or higher education refers to having completed $9+$ years of schooling.

${ }^{\mathrm{d}}$ Other includes skilled and unskilled labour, retired, other, unemployed and missing

${ }^{\mathrm{e}}$ Other includes other friend, casual acquaintance, commercial sex customer, relative and other.

${ }^{\mathrm{f}}$ Defined as men who reported one or more of the following reasons to justify hitting his partner: she does not do the housework to satisfaction; she disobeys him; she refuses sex; she asks about girlfriends; she takes a long time to get pregnant; she is childless; he suspects she is unfaithful; or he finds out she is unfaithful.

${ }^{g}$ STD symptoms include: deep lower abdominal pain, abnormal genital discharge, foul smell in genital area, excessive genital secretions, swelling of lymph nodes in genital area, itching in genital area, burning pain on micturition, deep pain during intercourse, genital ulcers or open sores, and others

community development, rather than individual development, affects levels of alcohol abuse. This is a question that could be addressed in subsequent analyses.

Partner's CAGE score was the foremost important predictor of alcohol abuse for women with partners, while for women without partners sexual risk factors were the main predictors. For men, both sexual risk factors and partner relations were associated with alcohol abuse. However, the direction of the association between sexual behaviours, partner relations and alcohol abuse cannot be determined on the basis of a cross-sectional study. Further longitudinal studies are warranted to assess the direction of these associations.

Despite the rough approximation of intimate partner violence, women with partners with CAGE scores 2-4 were more than twice as likely to report partner violence, although this association was non-significant in the adjusted model. This finding corresponded to the results of a study conducted in South Africa by Jewkes et al. (2003). Similarly, men who admitted to a number of reasons for hitting their partners were at greater risk of alcohol abuse. These associations point to a link between partner violence and alcohol abuse that should be further explored. Again, the direction of this association is unclear.

Risky sexual behaviour - specifically reporting more than two partners in the last year - was associated with alcohol abuse among men and women without a partner. 
Women without a partner, who reported current STD symptoms, were more likely to abuse alcohol than women who had no symptoms, although the association was not significant in the adjusted model. Similarly, men who tested positive for T. vaginalis were also more likely to report alcohol abuse than those who did not. These associations, together with multiple partners, point to an association between sexual risk and alcohol abuse that must also be further explored. However, unlike Kapiga et al. (2003), who found an association between HSV-2 and alcohol use in a group of bar and hotel workers in Moshi, there was no significant relationship between HSV-2 and alcohol abuse in any of the three groups in this study (the crude association was significant for men $(p=0 \cdot 02)$, but the adjusted was non-significant $(p=0 \cdot 20))$.

There are some limitations associated with this study. First, only women with sexual experience were included in the analyses. While consequently, the results may not be generalizable across all women, the vast majority of non-sexually experienced women were younger than age 25 and age was not linked to alcohol use. By analysing only sexually experienced women the analyses were able to look specifically at sexual practices and STDs that were presented in the literature as being important predictors of alcohol abuse. The sample of women was divided into those with and without partners to allow for a comparison with men (partners of the women). Women who reported never having had sex were primarily a subset of women without partners. Second, only 794 male partners were questioned out of a possible $1233(64 \%)$ and 788 men were analysed (64\%). It is unclear how the men who were not represented in the survey were different from those who were included. Men are generally more difficult to survey due to their mobility, particularly in this region, and therefore capturing $64 \%$ of all possible partners may be considered a reasonable response rate among the men. Finally, only men with a female partner were analysed and they may have different alcohol use patterns than single men.

In sum, a proportion of both men (23\%) and women (7-9\%) in Moshi in northern Tanzania reported alcohol abuse. While past studies may reflect the societal acceptance of such behaviour, it is important to explore further the association between risky sexual behaviours and alcohol abuse, and the potential risk of HIV infection and other STDs. While this study was exploratory in nature, it highlights the need to address alcohol abuse as a potential risk factor for unhealthy behaviours.

\section{Acknowledgments}

This research was funded by grant NIH/NICHD RO1 HD41202 to Ulla Larsen. Said Aboud and his colleagues from the Tanzania National Bureau of Statistics were responsible for the survey work. The authors would also like to acknowledge the contribution of the local interviewers, the data entry personnel and the laboratory staff.

\section{References}

Andrews, C. N., Kobusingye, O. C. \& Lett, R. (1999) Road traffic accident injuries in Kampala. East Africa Medical Journal 76, 189-194. 
Ao, T. T., Sam, N. E., Masenga, E. J., Seage, G. R. \& Kapiga, S. H. (2006) Human immunodeficiency virus type 1 among bar and hotel workers in northern Tanzania: the role of alcohol, sexual behavior, and herpes simplex virus type 2. Sexually Transmitted Diseases 33, 163-169.

Carlson, R. G. (1992) Symbolic mediation and commoditization: a critical examination of alcohol use among the Haya of Bukoba, Tanzania. Medical Anthropology 15, 41-62.

Central Intelligence Agency (CIA) (2004) The World Factbook 2004: Tanzania. Washington DC, http://www.cia.gov/cia/publications/factbook/geos/tz.html\#People.

Edwards, R., Unwin, N., Mugusi, F., Whiting, D., Rashid, S., Kissima J. et al. (2000) Hypertension prevalence and care in an urban and rural area of Tanzania. Journal of Hypertension 18, 145-152.

Ely, M., Hardy, R., Longford, N. T. \& Wadsworth, M. E. (1999) Gender differences in the relationship between alcohol consumption and drinking problems are largely accounted for by body water. Alcohol and Alcoholism 34, 894-902.

Ewing, J. A. (1984) Detecting alcoholism: the CAGE questionnaire. Journal of the American Medical Association 252(14), 1905-1907.

Ezzati, M., Lopez, A. D., Rodgers, A., Vander Hoorn, S. \& Murray, C. J. (2002) Selected major risk factors and global and regional burden of disease. Lancet 360, 1347-1360.

Fatoye, F. O. (2003) Psychosocial correlates of substance use amongst secondary school students in South Western Nigeria. East Africa Medical Journal 80, 154-158.

Henricson, C. G. (1992) Alcohol consumption in the Kilimanjaro area. In Kilonzo, G. P. \& Pitkanen, Y. T. (eds) Pombe: Report of Alcohol Research Project in Tanzania, 1988-90. University of Helsinki Institute of Development Studies, Helsinki, Finland.

Jewkes, R. (2002) Intimate partner violence: causes and prevention. Lancet 359, 1423-1429.

Jewkes, R. K., Levin, J. B. \& Penn-Kekana, L. A. (2003) Gender inequalities, intimate partner violence and HIV prevention practices: findings of a South African cross-sectional study. Social Science and Medicine 56, 125-134.

Kaaya, S. F., Kilonzo, G. P., Semboja, A. \& Matowo, A. (1992) Prevalence of substance abuse among secondary school students in Dar es Salaam. Tanzania Medical Journal 7, 21-24.

Kapiga, S. H., Sam, N. E., Bang, H., Ni, T., Quanhong, N., Trong, T. H. et al. (2007) The role of herpes simplex virus type 2 and other genital infections in the acquisition of HIV-1 among high-risk women in northern Tanzania. Journal of Infectious Diseases 195, 1260-1269

Kapiga, S. H., Sam, N. E., Shao, J. F., Masenga, E. J., Renjifo, B., Kiwelu, I. E. et al. (2003) Herpes simplex virus type 2 infection among bar and hotel workers in northern Tanzania: prevalence and risk factors. Sexually Transmitted Diseases 30, 187-192.

Kapiga, S. H., Sam, N. E., Shao, J. F., Renjifo, B., Masenga, E. J., Kiwelu, I. E. et al. (2002) HIV-1 epidemic among female bar and hotel workers in northern Tanzania: risk factors and opportunities for prevention. Journal of Acquired Immune Deficiency Syndrome 29, 409-417.

Kilonzo, G. P. (1990) Production and consumption of alcohol in Tanzania and alcohol-related accidents: an interdisciplinary study. In Maula, J., Lindblad, M. \& Tigerstedt, C. (eds) Alcohol in Developing Countries: Proceedings from a Meeting in Oslo, Norway, August 7-9, 1988. Nordic Council for Alcohol and Drug Research (NAD) (No. 18), Helsinki, Finland.

Kilonzo, G. P. (1992) Consumption of alcohol in Tanzania: country description. In Kilonzo, G. P. \& Pitkanen, Y. T. (eds) Pombe: Report of Alcohol Research Project in Tanzania, 1988-90. University of Helsinki Institute of Development Studies, Helsinki, Finland.

Kilonzo, G. P., Hogan, N. M., Mbwambo, J. K., Mamuya, B. \& Kilonzo, K. (2004) Pilot studies on patterns of consumption of nonindustrial alcohol beverages in selected sites, Dar es Salaam, Tanzania. In Haworth, A. \& Simpson, R. (eds) Moonshine Markets: Issues in Unrecorded Alcohol Beverage Production and Consumption. Brunner-Routledge, New York. 
Kilonzo, G. P. \& Maselle, A. Y. (1986) Substance misuse in Tanzania. Tanzania Medical Journal 3, 21-22.

Koenig, M. A., Lutalo, T., Zhao, F., Nalugoda, F., Kiwanuka, N., Wabwire-Mangen, F. et al. (2004) Coercive sex in rural Uganda: prevalence and associated risk factors. Social Science and Medicine 58, 787-798.

Koenig, M. A., Lutalo, T., Zhao, F., Nalugoda, F., Wabwire-Mangen, F., Kiwanuka, N. et al. (2003) Domestic violence in rural Uganda: evidence from a community-based study. Bulletin of the World Health Organization 81, 53-60.

Koss, M. P. \& Oros, C. J. (1982) Sexual Experiences Survey: a research instrument investigating sexual aggression and victimization. Journal of Consultative Clinical Psychology 50, 455-457.

Larsen, U., Mlay, J., Aboud, S., Ballard, R., Sam, N., Shao, J. \& Kapiga, S. (2007) Design of a community-based study of sexually transmitted infections/HIV and infertility in an urban area of Northern Tanzania. Sexually Transmitted Diseases 34(1), 20-24.

Luginaah, I. \& Dakubo, C. (2003) Consumption and impacts of local brewed alcohol (akpeteshie) in the Upper West Region of Ghana: a public health tragedy. Social Science and Medicine 57, 1747-1760.

McCall, M. (1996) Rural brewing, exclusion, and development policy-making. Gender and Development 4, 29-38.

McFarlane, J., Parker, B., Soeken, K. \& Bullock, L. (1992) Assessing for abuse during pregnancy: severity and frequency of injuries and associated entry into prenatal care. Journal of the American Medical Association 267, 3176-1378.

Maula, J. (1990) Research in the production and consumption of alcohol in Tanzania: some preliminary reflections. In Maula, J., Lindblad, M. \& Tigerstedt, C. (eds) Alcohol in Developing Countries: Proceedings from a Meeting in Oslo, Norway, August 7-9, 1988. Nordic Council for Alcohol and Drug Research (NAD) (No. 18), Helsinki, Finland.

Mphi, M. (1994) Female alcoholism problems in Lesotho. Addiction 89, 945-949; discussion 951-960.

Nikander, P., Honkanen, R., Huttunen, P., Kilonzo, G. P. \& Pitkanen, Y. T. (1992) Role of alcohol in injuries treated in the Muhimbili Medical Centre casualty department, Dar es Salaam, Tanzania. In Kilonzo, G. P. \& Pitkanen, Y. T. (eds) Pombe: Report of Alcohol Research Project in Tanzania, 1988-90. University of Helsinki Institute of Development Studies, Helsinki, Finland.

Odero, W. (1998) Alcohol-related road traffic injuries in Eldoret, Kenya. East Africa Medical Journal 75, 708-711.

Odero, W., Garner, P. \& Zwi, A. B. (1997) Road traffic injuries in developing countries: a comprehensive review of epidemiological studies. Tropical Medicine and International Health 2, 445-460.

Odero, W. \& Zwi, A. B. (1997) Drinking and driving in an urban setting in Kenya. East Africa Medical Journal 74, 675-679.

Perdrix, J., Bovet, P., Larue, D., Yersin, B., Burnand, B. \& Paccaud, F. (1999) Patterns of alcohol consumption in the Seychelles Islands (Indian Ocean). Alcohol and Alcoholism 34, 773-785.

Rijken, T., Velema, J. P. \& Dijkstra, R. (1998) Alcohol consumption in the rural population of Misungwi subdistrict in Mwanza Region, Tanzania. Journal of the Studies of Alcohol 59, 146-151.

Room, R. V. (2002) Alcohol and the Developing World: A Public Health Perspective. Finnish Foundation for Alcohol Studies in collaboration with World Health Organization, Helsinki.

Sa, Z. \& Larsen, U. (2007) Gender inequality and HIV-I infection among women in Moshi, Tanzania. Journal of Biosocial Science (in press). 
Secretary of Health and Human Services (HHS) (2000) 10th Special Report to the US Congress on Alcohol and Health: Highlights from Current Research. US Department of Health and Human Services, Washington, DC.

Setel, P. W. (1999) A Plague of Paradoxes: AIDS, Culture and Demography in Northern Tanzania. University of Chicago Press, Chicago and London.

Shayo, N. B., Kamala, A., Gidamis, A. B. \& Nnko, S. A. (2000) Aspects of manufacture, composition and safety of orubisi: a traditional alcoholic beverage in the north-western region of Tanzania. International Journal of Food Sciences and Nutrition 51, 395-402.

Siegfried, N., Parry, C. D., Morojele, N. K. \& Wason, D. (2001) Profile of drinking behaviour and comparison of self-report with the CAGE questionnaire and carbohydrate-deficient transferrin in a rural Lesotho community. Alcohol and Alcoholism 36, 243-248.

Straus, M. (1990) The conflict tactics scale revisited. In Straus, M. A. \& Gelles, R. (eds) Physical Violence in Families. Transaction Publishers, New Brunswick, NJ.

Suter, P. M. (2004) Alcohol, nutrition and health maintenance: selected aspects. Proceedings of the Nutrition Society 63, 81-88.

Tusekwa, A. B., Mosha, T. C., Laswai, H. S. \& Towo, E. E. (2000) Traditional alcoholic beverages of Tanzania: production, quality and changes in quality attributes during storage. International Journal of Food Science and Nutrition 51, 135-143.

World Health Organization (WHO) (2001) Global Status Report on Alcohol. WHO, Geneva.

World Health Organization (WHO) (2002) World Health Report 2002. WHO, Geneva. 